\title{
Genomics boosts brain-cancer work
}

\author{
Molecular findings start to open up avenues of diagnosis and treatment.
}

Few diseases are more intractable than brain cancer. Many factors make it difficult to treat, including the sensitivity of the brain, the complexity of the disease - catch-all diagnoses can obscure differences between tumour types and the small number of patients, which limits the power of clinical trials and makes them less attractive to large drug companies. "Until recently, brain cancer was considered almost too challenging to work on," says Paul Mischel, a neuropathologist at the David Geffen School of Medicine in Los Angeles.

But researchers say that the field is gaining momentum from cancer genomics. Molecular approaches are improving understanding of the disease and pointing to possible new treatments.

"Brain cancer is a disease that needs some new breakthroughs, and this is creating a lot of energy," says Susan Fitzpatrick, vice-president of the James S. McDonnell Foundation in St Louis, Missouri, which funds brain-cancer research. "I detect a fair amount of optimism, a sense that there are more avenues open that may lead to something positive."

This week, for example, researchers led by Neil Hayes of the University of North Carolina in Chapel Hill combined geneexpression data with DNA mutation data from 200 samples of glioblastoma ${ }^{1}$, the most common malignant brain cancer in adults. They found that the tumours fell into four groups broadly similar to some proposed earlier. Each group has distinctive molecular markers and, potentially, different vulnerabilities that could be exploited for treatment.

Something positive is sorely needed for glioblastoma, which kills most patients within a year. Standard treatment includes surgery to remove the bulk of the tumour, followed by radiation and treatment with the drug temozolomide. Last May, the US Food and Drug Administration approved the biological drug bevacizumab, sold by Genentech of South San Francisco as Avastin, for use in patients whose tumours have returned after surgery.

But molecular data could help to refine the approach. In 1998, a group led by David Louis of Massachusetts General Hospital in Boston showed that patients who had gliomas - the category of brain cancer that includes glioblastomas - that had lost portions of chromosomes 1 and 19 were more likely to respond to chemotherapy, and survived longer after their treatment with- out their cancer recurring, than did those who retained the chromosome segments ${ }^{2}$. Genetic tests for these mutations are now used to predict the course of patients' disease and to guide treatment decisions.

\section{Treasure troves}

Such findings led the US National Cancer Institute to set up the Repository of Molecular Brain Neoplasia Data (REMBRANDT) in 2004. In 2006, the National Institutes of Health's Cancer Genome Atlas (TCGA) chose glioblastoma as one of the first three cancers it would subject to in-depth genetic and molecular characterization. In 2008, the group published its initial

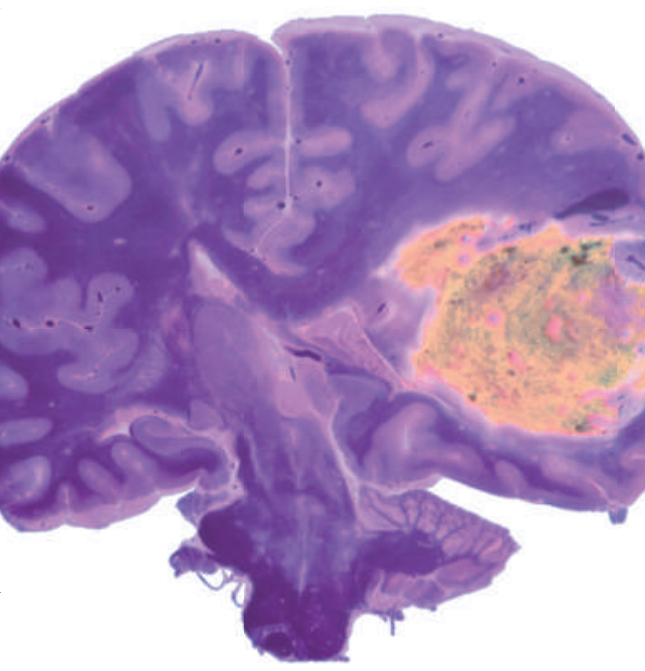

Brain tumours are notoriously hard to treat.

genomic analysis of the disease ${ }^{3}$, at around the same time that a group based at Johns Hopkins University in Baltimore, Maryland, published its genomic analysis of the cancer ${ }^{4}$. Such work is galvanizing the field, researchers say.

"What's come out of these large studies is an extraordinary set of publicly available resources that are absolutely key for investigators to use for discovery, and I think the value of that is almost incalculable," Louis says.

This week's announcement of the four subtypes of glioblastoma ${ }^{1}$ also involved TCGA researchers. They found that $97 \%$ of tumours in the 'classical' subtype carried extra copies of the EGFR gene, and most had higher than normal expression of EGFR — whereas the gene TP53, which is often mutated in glioblastoma, was rarely mutated in this subtype.

Researchers are already using such subtypes to define classes of patients who might benefit most from targeted therapies in clinical trials, but the new work adds detail about the specific genomic characteristics. For instance, in 2006 neuropathologist Ken Aldape of the University of Texas M. D. Anderson Cancer Center in Houston, and his colleagues, used gene-expression data to devise a classification scheme $e^{5}$ for gliomas that is now being tested as part of a clinical trial of bevacizumab in patients newly diagnosed with glioblastomas, to see which tumour types respond best to the treatment.

Defining patient subgroups could ensure that researchers don't overlook drugs that help only a fraction of patients. "We may be throwing away active drugs because we're seeing minimal response rates, but it may be that the biology never gave that drug a chance, because it was only destined to work in $5 \%$ of patients," says Howard Fine, chief of the National Cancer Institute's Neuro Oncology Branch in Bethesda, Maryland.

Genomic information may already be revealing new drug targets. For instance, the Johns Hopkins study ${ }^{4}$ showed that $12 \%$ of glioblastomas had a mutation in a gene called $I D H 1$, and a subsequent study ${ }^{6}$ found that $70 \%$ of all gliomas have this mutation. Last year, a team led by researchers at the biotech company Agios Pharmaceuticals in Cambridge, Massachusetts, reported that mutated IDH1 enzymes boost the production of $R(-)$-2-hydroxyglutarate ${ }^{7}$, a metabolite linked to brain cancer. The firm is now looking for drugs that dampen the activity of the mutated enzyme.

Scientists are also designing clinical trials of drugs that aim to hit targets identified in previous molecular studies, such as pathways involving the PTEN gene and the proteins Notch, MAPK and EGFR.

None of these trials is guaranteed to yield treatments, but, as Fine says, brain-cancer researchers now have a variety of possible targets to aim for, whereas before they had nothing. "For once," he says, "we have a way to go."

Erika Check Hayden

1. Verhaak, R. G. W. et al. Cancer Cell 17, 98-110 (2010).

2. Cairncross, J. G. et al. J. Natl Cancer Inst. 90, 1473-1479 (1998).

3. The Cancer Genome Atlas Research Network Nature 455, 1061-1068 (2008)

4. Parsons. D. W. et al. Science 321, 1807-1812 (2008)

5. Phillips. H. S. et al. Cancer Cell 9, 157-173 (2006).

6. Yan, H. et al. N. Engl. J. Med. 360, 765-773 (2009)

7. Dang, L. et al. Nature $462,739-744$ (2009). 